\title{
Internet of Hybrid Energy Harvesting Things
}

\author{
Ozgur B. Akan, Fellow, IEEE, Oktay Cetinkaya, Student Member, IEEE, Caglar Koca, Student \\ Member, IEEE, Mustafa Ozger, Student Member, IEEE
}

\begin{abstract}
Internet of Things (IoT) is a perfect candidate to realize efficient observation and management for Smart City concept. This requires deployment of large number of wireless devices. However, replenishing batteries of thousands, maybe millions of devices may be hard or even impossible. In order to solve this problem, Internet of Energy Harvesting Things (IoEHT) is proposed. Although the first studies on IoEHT focused on energy harvesting as an auxiliary power provision method, now completely battery-free, self-sufficient systems are envisioned. Taking advantage of diverse sources that the application areas in Smart Cities offer helps us to fully appreciate the capacity of energy harvesting. In this way, we address the primary shortcomings of IoEHT; availability, unreliability and insufficiency by the Internet of Hybrid Energy Harvesting Things (IoHEHT). In this work, we survey the various energy harvesting opportunities, propose an hybrid energy harvesting system and discuss energy and data management issues for battery-free operation. We also point out to hardware requirements and present the open research directions for different network layers specific to Internet of hybrid energy harvesting things for Smart City concept.
\end{abstract}

Index Terms-Hybrid Energy Harvesting, Wireless Networks, Internet of Things, Smart Cities.

\section{INTRODUCTION}

Enhanced management of cities brings a new paradigm, named as Smart Cities [1], [2], which achieves environment sensing and better utilization of city resources. Particular application areas of Smart Cities are intelligent transport systems, smart grid, smart home, smart agriculture and structural health [3]. The realization of them requires utilization of cutting edge technologies such as the Internet of Things (IoT). Sensing and controlling features of the IoT are keys to this realization. Using IoT, the physical world can be observed, and information related to the surroundings is gathered, such that the physical world is digitized. Using IoT technology, we can access to this digitized world via the Internet connection, and move one step closer to the Smart City concept [4], [5].

In order to achieve continuous monitoring and control, an auxiliary or even a completely distinct power source should be equipped to the sensors. However, even this option may or may not be applicable in some cases mostly due to size constraints or design restrictions. Hence, energy harvesting methods come into prominence to alleviate the problems of energy-constrained wireless networks by exploiting a stray source or converting energy from one form to another [6], [7].

Ozgur B. Akan is with Internet of Everything (IoE) Group, Electrical Engineering Division, Department of Engineering, University of Cambridge, CB3 0FA Cambridge, UK (e-mail: oba21@cam.ac.uk).

O. Cetinkaya, C. Koca and M. Ozger are with the Next-generation and Wireless Communications Laboratory (NWCL), Department of Electrical and Electronics Engineering, Koc University, Istanbul, 34450, Turkey (e-mail: \{okcetinkaya13, cagkoca, mozger\}@ku.edu.tr).
There are numerous potential alternatives to collect energy, but their availability depends on the environmental variables, ambient parameters, or other time-varying and highly random external factors. The ongoing limits on the power extraction capabilities force wireless devices for an energy trade-off between proper system operation and the desired network lifetime, whereby an upper bound is placed on the communication reliability. Due to this reason, hybrid energy scavenging approaches possess a great potential to extend the lifetime of wireless devices by operating in a complementary manner. A power supply fed by multiple available sources will eventually enhance the overall functionality, reliability, and efficiency of both the system and communication [8]-[13].

The hybrid energy harvesting wireless smart nodes sense the parameters of interest, process the collected data, and report the resulting information to a base station/coordinator/gateway over an Internet connection where the conditions of application area are monitored, stored, and relevant authorities are alerted.

Energy modeling is crucial in any harvesting mechanism, as optimal transmission policy directly depends on the energy model. Hybrid energy harvesting enhances energy availability, and therefore, improves the energy model of the system. Moreover, in order to survive in the most dire circumstances of Smart Cities, data management protocols, specific to IoHEHT are needed. Furthermore, hybrid energy harvesting proposal for IoT-enabled Smart Cities requires novel approaches in each network layer to overcome the challenges posed by IoT and Smart Cities to enable seamless operation. Hence, we lay the foundations of battery-free IoHEHT networks.

In this paper, we first present existing energy harvesting (EH) techniques, and then propose a new EH framework. It is called hybrid energy harvesting, and copes with randomness of harvestable resources by utilizing different $\mathrm{EH}$ methods together. Furthermore, an applicable design for a hybrid EH sensor system is presented. We also model energy and data, and study mathematically the decrease of harvestable energy variance by the hybrid approach. We test our new EH framework with a simulation of a communication scenario, showing that hybrid energy harvester can achieve lower drop rates for the same reporting frequency. We propose a model for energy and data queue management according to the proposed $\mathrm{EH}$ method. Open issues and problems are discussed for each layer in IoT networks utilizing hybrid EH.

The remainder of this paper is organized as follows. First, we commence with a literature review of the existing energy harvesting techniques. Then, we extend our study in Section III to basic principles of hybrid energy harvesting systems including their basis, main constraints and applicable procedures in the IoT domain. This is followed by the performance analysis of IoHEHT by investigating energy and data models 
Table I: Comparison of the existing energy harvesting techniques [17].

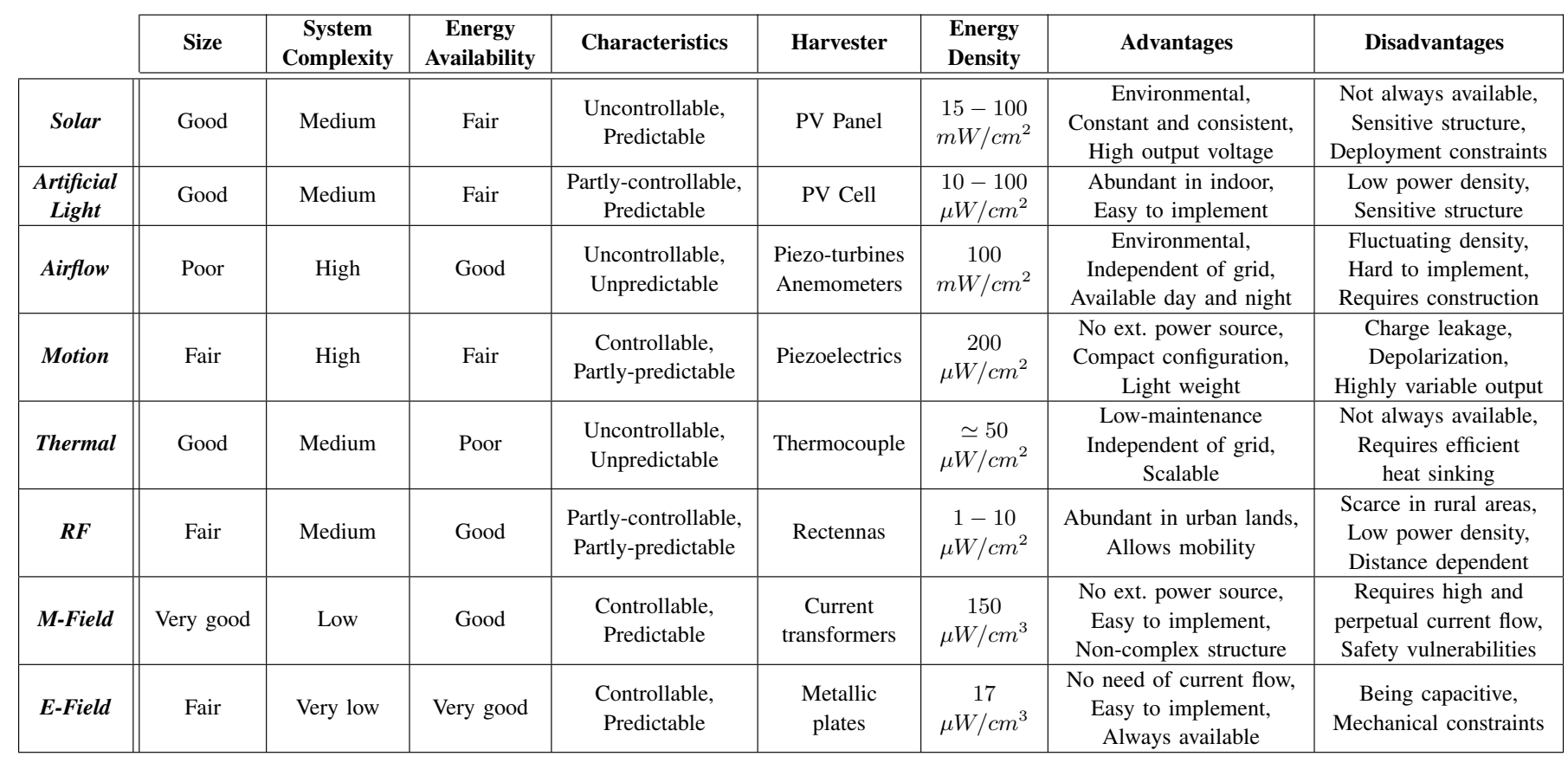

and applicable transmission policies in Sections IV and V, respectively. We address the applicable transmission policies as well as open research directions for different network layers specific to the IoHEHT procedures in Section VI. Finally, we conclude our discussion in Section VII.

\section{EXIsting EnERgy HARVESTING TeChNiQUeS}

When a small-scale industrial, medical, and/or educational facility is envisioned, the continuity of communication is of paramount importance. Any interruption or failure may not be tolerated due to the vitality of the task that is being fulfilled. This fact one again reveals the need for a complementary procedure, i.e., a hybrid energy harvesting architecture. Existing energy sources can be broadly divided into four groups as light, heat, motion, and electromagnetic (EM) radiation, in which availability, controllability, and predictability of these sources determine the models and specifications of the harvesting procedures that are going to be employed [6], [7].

By regarding this separation, the frequency of preference, and the motivation of our proposal some leading energy harvesting methods are discussed below, and a detailed comparison is illustrated in Table I.

\section{A. Light Energy Harvesting}

Energy harvesting form light sources is a well-established method of power provision that gathers energy from ambient lights, either from sun or artificial light sources, with respect to a phenomena called as photo-voltaic (PV) effect [6], [7]. In outdoor, for the monitoring of overhead power lines, solar cell inlaid photo-voltaic panels are used to convert solar energy into electricity [14], [15]. For indoor applications, specialized photo-voltaic materials, which are better suited for diffused lights, are employed for taking advantage of the light emitted from ambient elements. Even though the PV modules are getting cheap, easy to use and efficient, due to the dramatic fluctuations on the output power, large surface area requirements, inoperability at night and ongoing installation and maintenance costs, their use in mission critical applications is limited [16]. However, for intermittent reporting allowed ambient sensing and management services of Smart Home/Building architectures IoT-capable light EH sensor nodes are intensively preferred.

\section{B. Kinetic Energy Harvesting}

Kinetic energy harvesting $(\mathrm{KEH})$ is the conversion of ambient mechanical energy into electric power. Wind turbines, anemometers and piezoelectric materials are being developed to attain energy from highly random and unpredictable motion variations driven by external factors [6], [7].

$\mathrm{KEH}$ is frequently preferred in indoor and outdoor domain, as a variety of sources can be conveniently exploited to drive low power consumptive wireless autonomous devices. In outdoor, airflow operated IoT-capable sensor nodes are satisfactorily utilized for remote monitoring of the spaced apart grid assets. Similarly, for less power requiring wireless devices, any source of motion variation offers sufficient solutions for low duty-cycled communications. However, designing a generalized harvesting system especially for vibrating sources is an ongoing challenge. Since the conversion efficiency highly varies with the resonant frequency of the vibration, a specialized design for each source may be necessary [14]-[17].

\section{Thermal Energy Harvesting}

Thermal energy harvesting, i.e., thermoelectric generation (TEG), is simply based on converting temperature gradients into utilizable electric power with respect to the Seeback Effect 
occurred in semiconductor junctions [7]. TEG is an innate power provision technique for Smart Grid communications, in which temperature swings between the power line and the environment is used to extract energy. In small scale, peltier/thermoelectric coolers and thermocouples are widely used for building delay-tolerant wireless indoor networks [17]. Although harnessing power from temperature gradients sounds promising, there is a fundamental limit, namely Carnot limit, to the maximum efficiency at which energy can be harvested from a temperature difference [7], [14], [16].

\section{Electromagnetic Energy Harvesting}

EM energy harvesting includes collecting RF signals emitted from base stations, network routers, smartphones, and any other sources by using large aperture power receiving antennae, and converting the attained waves into utilizable DC power [6], [7]. Their performance depends strongly on the RF to DC conversion efficiency and the amount of power received by the antennae. Although this method is a reliable solution unaffected by the environmental variables, providing relatively low power densities, necessitating close deployment to the network transmitters, and requiring additive components such as filters and voltage multipliers can be counted as its main shortcomings [14]-[17]. Moreover, in case the nodes are sparsely deployed, available energy to be harvested may be too low, which might limit the use of EM energy harvesting. Due to the abundance of EM propagation in urban areas, RF energy harvesting is mostly preferred to operate IoT-assisted Smart City services.

\section{E. Magnetic-field Energy Harvesting}

M-field energy harvesting is based on coupling the field flow around the $\mathrm{AC}$ current carrying conductors that is clamped by current transformers (CT) [15], [18]. This technique is able to provide an adequate rate of continuous power so long as current flow in the line is sufficient. As the amount of current on power distribution level is considered, M-field EH stands as the best candidate for the energization of high power requiring IoT networks. However, gathering energy from a high current carrying asset in close proximity to the harvester in a safe way is still a challenging issue. To mitigate the safety concerns, M-field-based methods need to be equipped with advanced protection and control mechanisms. This issue compels their utilization in terms of circuit complexity and implementation flexibility [17].

\section{F. Electric-field Energy Harvesting}

According to the basics of electrostatics, any conductive material energized at some voltage level emits electric field. In $\mathrm{AC}$, time varying field results in a displacement current, whereby the E-Field induced electric charges are dispatched and collected in storing element. As the accumulated energy is gathered from the surrounding field, this method is named Efield energy harvesting (EFEH) [15], [17], [19], [20]. E-field is the only source that is neither intermittent nor dependent on the load [21]. As the voltage and the frequency are firmly

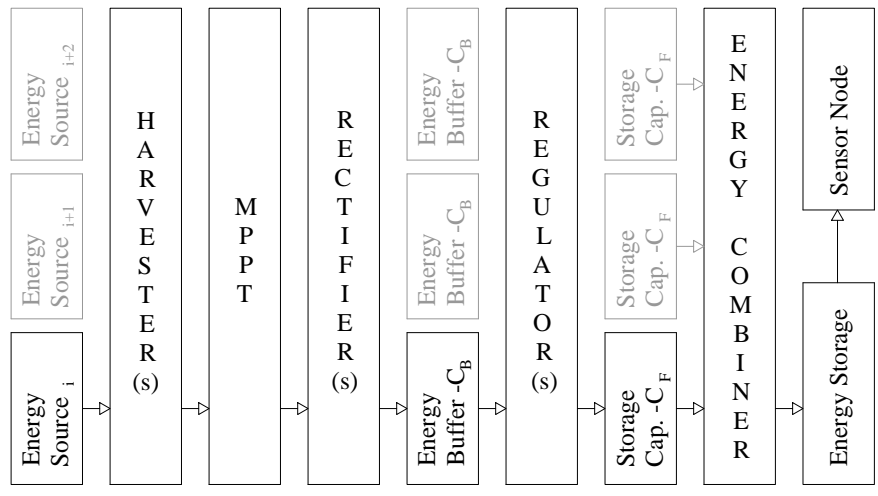

Figure 1: An applicable design for a hybrid energy harvesting sensor system. Note that, gray blocks represent sub-systems of modular design.

regulated and exactingly maintained, the E-field is therefore stable and predictable in its behavior. Thus, it can be referred as the most promising way to compose long-term and selfsustainable IoT networks notwithstanding the ambient factors.

\section{HYBRID ENERGY HARVESTING}

All the energy harvesting methods discussed above are used in such applications like wireless networking and remote monitoring. However, availability of natural sources affects the power density, and durability of their operation dramatically. To exemplify, solar energy is extremely sensitive to the environment, i.e., it is only exploitable during daytime. The very same problem is also seen in non-environmental sources, in which the harvesting performance is highly threatened by the randomness of the ambient variables, although the sources are partly-controllable in general. As all available techniques of EH depend strongly on environmental conditions, grid-based variables or any other uncontrollable parameters, hybrid solutions become even more important for sustaining information and/or time critical communications [8]-[11].

In order to obtain the best performance achievable, a twostaged performance maximization process is recommended for hybrid energy harvesting systems [12]. Fig. 1 depicts such a possible architecture. In the first stage, the harvesters are required to maintain their operation as collecting maximum energy possible from the available sources. For that purpose, such approaches like maximum power point tracking (MPPT) are developed for compensating inconsistencies and accordingly maximizing the scavenging efficiencies. As each harvesting method has an optimal operation point that varies with the amount of harvastable energy, MPPT procedures should be capable of real-time tracking, and highly responsive to any change in sources' conditions. In addition to this power extraction related approach, further effort should be focused on how to convert, and transfer the gathered energy as efficiently as possible, since the scavenged energy is still quite low and highly time-varying.

The second stage includes efficient combination and management of the exploited sources. As energy is gathered simultaneously from distinct harvesters, an energy combiner is required to accumulate the individual contributions of each 


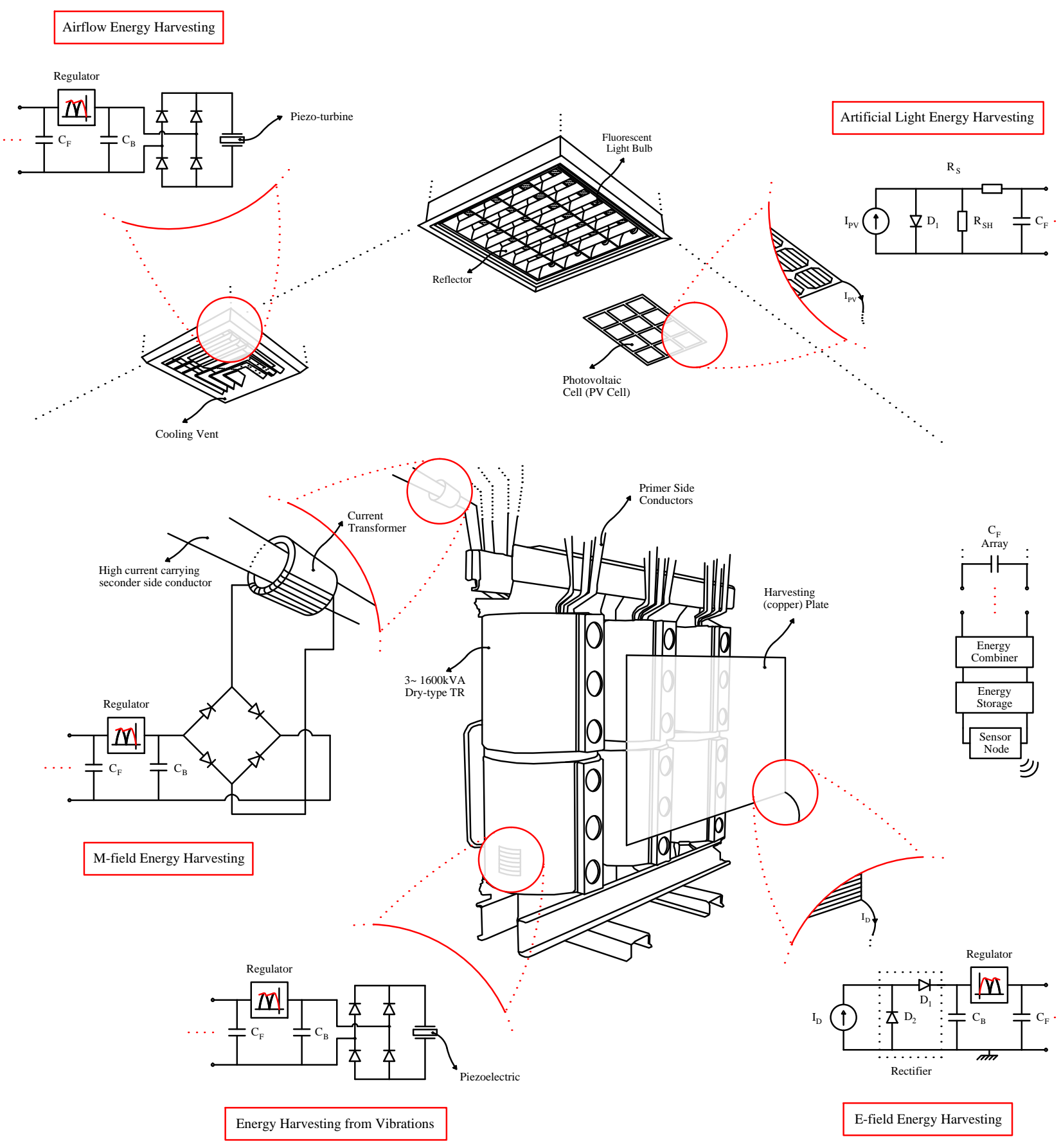

Figure 2: Representative drawing of the proposed hybrid energy harvesting architecture for IoT.

system in a storage whereby the overall energy is delivered to a wireless device, i.e., sensor node, autonomously. The combiner needs a modular design that supports a variety of EHs and their corresponding circuitries to be attached as subsystems. Note that, the eventual standardization of IoT ease the modular design of such systems. In this way, the connection of complementary sources is ensured in a very straightforward manner at the expense of few components. For such an architecture, an adaptive connection mechanism is needed to isolate the harvesters from each other, such that undesired interferences are prevented, i.e., charging each other instead of the storing element. [12], [13]. In addition, combining sources in close proximity with each other using this circuitry autonomously allows charge conveyance when the collected energy is high enough for transmission, and switch off the sensory circuit when the voltage of the storage drops beyond a certain threshold [8], [22]. This operation not only prevents redundant and undesired discharge of the storage to $0 \mathrm{~V}$, but also allows more frequent data transmission by shortening the charging time [17]. As the energy collected by different sources can be combined in a universal depository, i.e., energy storage in Fig. 1, it can also be kept separately in sub-level 
buffers to supply different loads or sensors. This operation, i.e., supporting various energy harvesting techniques as well as energy storing systems points out to a newly-emerging topic, namely multi-input multi-output (MIMO) energy harvesting (MIMO-EH). However, MIMO-EH is still at its infancy.

Fig. 2 illustrates the physical model depiction of a representative IoT scenario for a transformer, a pillar of the Smart Grid infrastructure, powered by hybrid energy harvesting. The hybrid energy harvesting node equipped with specialized sensors such as; light, temperature, humidity, and presence, is envisioned to observe the parameters of both the room and transformer, process the extracted data, and notify upper level authorities over the Internet for decision-making procedures. With Internet connectivity, preclusive actions can be simultaneously fulfilled against any intruder and/or unexpected variations in medium parameters. The lifetime of the IoT network can be further prolonged by harvesting multiple-sources in the vicinity of the environment, which guarantees interruptionfree operation of the transformer. In this figure, there are five distinct sources of interest for energy provision. The nature of these sources do differ immensely which inevitably affects the characteristics of the energy gathered. In other words, certain harvesting methods require rectification, regulation and/or conversion processes due to their high voltage low current AC output, while some others need only one or two of these procedures. However, in general, the circuits employed after power acquisition stage can be referred as roughly similar to each other. From Fig. 1 and Fig. 2, the diodes, i.e., rectifiers, are for both rectifying the alternating current, and preventing the harnessed energy from back feeding. The converted energy is first stored in an energy buffer $C_{B}$ before regulation. As the name suggests, regulators ensure delivering suitable and stable voltage supply to the other parts of the circuit. They can also be supported by additive smoothing and charge control circuits for enhanced performance. The regulated energy is then accumulated in a storage capacitor $C_{F}$, to be combined with the output of other distinct sources/harvesters. The energy combined is stored in a quick-charged, long-lasting, and high power-condensed super capacitor to boost longevity. DC-toDC converters, which are not shown in figures, can also be employed to adjust the voltage output of the energy storage to ensure proper operation of the attached load, i.e., sensor node. Overall performance of such a system depends on the efficiency of the equipped components and employed procedures, as well as duty cycle of the sensor node; and the protocol stack.

Harvesting energy from several sources simultaneously acts as an insurance in case of energy scarcity. In other words, each harvester mechanism is partly responsible for energy acquisition, and they complement each other when any of them fail to provide enough power in the absence and/or insufficiency of the exploited source. As this operation increases the overall system reliability, it becomes possible to run the wireless devices as if they have a constant energy source like batteries. By using hybrid energy harvesting-enabled Internetcapable sensors, sensory data can be remotely observed by a network coordinator, and necessary actions can be directed over Internet. This better supported operation will eventually help to achieve more reliable, responsive, and inter-operable IoT networks for advanced Smart City services. Following sections are investigating this proposition and questioning the availability of transmit power maximization with respect to hybrid energy profile of the universal harvesting system.

\section{ENERGY AND DATA MODELING}

A crucial aspect of energy harvesting is profiling the energy. Any EH system should be designed specific to the energy profile of the resource to be exploited. The most common assumption in energy profiling is offline profiling, where it is assumed that the energy availability and data transmission requirements are known beforehand. In this case, network design should be optimized to the expected harvestable energy, i.e., any design powered by solar energy harvesting should keep in mind that there will be no harvestable energy at night. In case such an information does not exist, harvesters should adjust to the energy and data arrivals, i.e., online profile. Such designs need to handle more uncertainties in the energy arrivals.

Whether an offline energy profile exists or not, harvester design must consider two principles: Energy causality and Data causality. Energy causality implies energy cannot be used before it is harvested. Similarly, data causality implies any data that has not arrived cannot be transmitted.

In order to model the harvested energy and energy required for transmission, we use energy line and data line, respectively. Energy line, $e(t)$ is the total amount of energy harvested until time $t$, while data line, $d(t)$ is the total amount of energy required to process all arrived data packages until time $t$, i.e.,

$$
\begin{gathered}
e(t)=\int_{0}^{t} P_{\text {harvested }}\left(t^{\prime}\right) \mathrm{d} t^{\prime} \\
d(t)=\int_{0}^{t} P_{\text {used }}\left(t^{\prime}\right) \mathrm{d} t^{\prime}
\end{gathered}
$$

where $P_{\text {harvested }}(t)$ and $P_{\text {used }}(t)$ are the harvested and exhausted power between $t$ and $t+\Delta t$, respectively. Note that if the system is supplied with a battery, $e(t)$ should be a constant line. Also note that, any packet arriving to the queue causes an increase in the data line and any packet dropped from the queue causes a drop in the data line. Total available energy in the system is

$$
E_{\text {available }}(t)=\min (C, e(t)-d(t))
$$

where $C$ is the total energy storage capacity. Since $E_{\text {available }}$ cannot be negative, if minimum amount of energy required to process the arrived data, exceeds total harvested energy, i.e., some data must be dropped. The area lying between energy line and data line is called feasible energy tunnel. Energy line, data line, feasible energy tunnel and storage element size for a generic harvesting scenario is shown in Fig. 3(a).

Examining Fig. 3(a), we realize that any energy allocation policy must lie between the total harvested energy and minimum amount of energy required to process all data. Furthermore, an optimal policy should minimize the storage overflow while maximizing the transmission rate. Such an optimal energy allocation policy is proven to be the shortest 


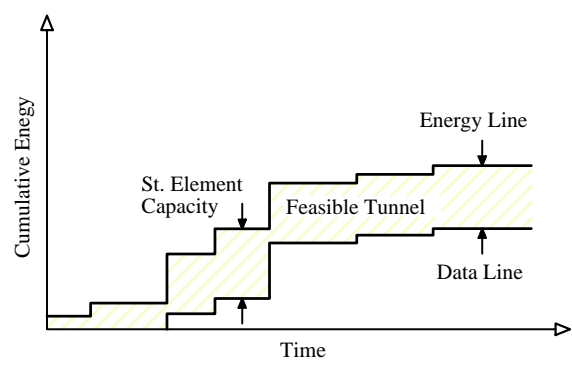

(a)

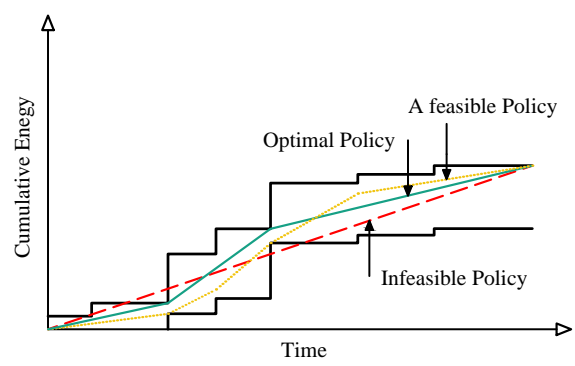

(b)

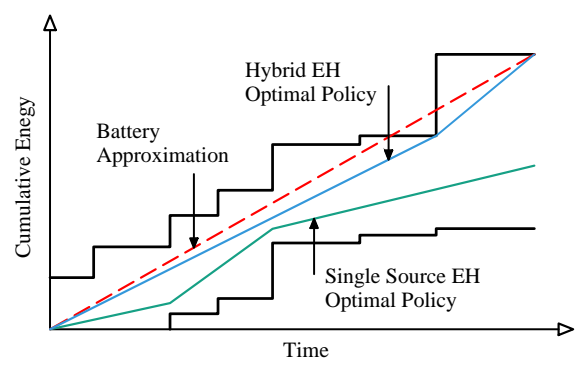

(c)

Figure 3: (a) Main components of energy and data models; (b) Optimal policy, feasible policy and infeasible policy for single source energy harvesting; (c) Optimal policy for single source energy harvesting; optimal policy for hybrid energy harvesting and battery approximation.

path connecting the start and end points [23]. Possible and optimal energy allocation policies for a generic harvesting scenario are shown in Fig. 3(b).

Hybrid energy harvesting enables us to diversify energy sources. An obvious benefit of hybrid energy harvesting in offline energy profiling is the increase in the harvested energy. Increased available energy expands the feasible energy tunnel, such that the optimal energy allocation policy can be enhanced. If a large enough storage element is available, optimal policy may acts as if the system is battery powered, i.e., the straight line connecting the start and finish points. Note that, in order to take full advantage of hybrid energy harvesting, a larger storage element compared to single source energy harvesters should be used. Otherwise, storage overflows may diminish the system performance. The effects of hybrid energy harvesting is presented in Fig. 3(c). Note that the optimal policy in Fig. 3(b), depicted as the single source EH optimal policy in Fig. 3(c), is inferior to the hybrid EH optimal policy as it is further away from battery approximation.

In case the energy profile of the sources is not known well, i.e., the sources are unpredictable; in addition to increasing the overall energy available for transmission, hybrid energy harvesting boosts reliability. Using different energy sources, regardless of their variance, reduces the overall variance and increases the total amount of the harvestable energy. Both additional energy and reduced variance of the harvestable energy are helpful in increasing the transmission rate and reducing the packet drops.

The full advantage of hybrid energy harvesting can only be appreciated if more than one sensor is connected to a single transmitter. In other words, we pool the total harvestable energy from $n$ sources and drive $n$ sensors using energy from this pool. Such a system is depicted in Fig. 6. Here, we will show that hybrid harvesting of $n$ sources to power $n$ sensors is more reliable than single source harvesting.

Theorem 1. Assume that we need to run $n$ sensors, i.e., $S_{i}$, in close proximity with $n$ exploitable resources, i.e., $R_{i}$, with average harvestable power $P_{i}$ and variance $\sigma_{i}$. Hybrid energy harvesting reduces the variance of all sources $R_{i}$ satisfying

$$
\sigma_{i}^{2}>\left(\frac{P_{i}}{\sum_{j=1}^{n} P_{j}}\right)^{2} \sum_{j=1}^{n} \sigma_{j}^{2}
$$

Proof. Assume the average power output of $R_{i}$ is provisioned hybridly by all sources, i.e.,

$$
R_{H_{i}}=\frac{P_{i}}{\sum_{j=1}^{n} P_{j}} R_{i}
$$

Variation of $R_{H_{i}}$ is calculated as

$$
\begin{aligned}
\operatorname{Var}\left(R_{H_{i}}\right) & =\operatorname{Var}\left(\frac{P_{i}}{\sum_{j=1}^{n} P_{j}} \sum_{j=1}^{n} R_{j}\right) \\
& =\left(\frac{P_{i}}{\sum_{j=1}^{n} P_{j}}\right)^{2} \sum_{j=1}^{n} \sigma_{j}^{2} .
\end{aligned}
$$

Using (7), we can easily see that $\operatorname{Var}\left(R_{i}\right)<\operatorname{Var}\left(R_{H_{i}}\right)$ if (4) holds.

Corollary 1. Hybrid energy harvesting provides energy provision with reduced variance for at least one sensor node

Proof. Assume the variance of the exploitable resources are $\sigma_{1}^{2} \leq \sigma_{2}^{2} \leq \cdots \leq \sigma_{n}^{2}$. The variance of the largest variance resource, $R_{n}$, is clearly less than $\operatorname{Var}\left(R_{H_{n}}\right)=$ $\frac{P_{n}}{\sum_{j=1}^{n} P_{j}} \sum_{j=1}^{n} \sigma_{j}^{2}$.

Corollary 2. Hybridization of two resources boost the variance of the average power output for both sensors if

$$
\left(\left(\frac{P_{2}}{P_{1}}\right)^{2}+2 \frac{P_{2}}{P_{1}}\right)>\frac{\sigma_{2}^{2}}{\sigma_{1}^{2}}>\left(\left(\frac{P_{1}}{P_{2}}\right)^{2}+2 \frac{P_{1}}{P_{2}}\right)^{-1}
$$

Proof. Using the variance formula in Eq. (7) for two sources, and solving for the region satisfying $\sigma_{H_{i}}^{2}<\sigma_{i}^{2}$,

$$
\begin{gathered}
\sigma_{1}^{2} \geq \frac{P_{1}^{2}}{\left(P_{1}+P_{2}\right)^{2}}\left(\sigma_{1}^{2}+\sigma_{2}^{2}\right) \\
\sigma_{2}^{2} \geq \frac{P_{2}^{2}}{\left(P_{1}+P_{2}\right)^{2}}\left(\sigma_{1}^{2}+\sigma_{2}^{2}\right)
\end{gathered}
$$

for $R_{1}$ and $R_{2}$ respectively. Solving Eq. (9) and (10) simultaneously we obtain Eq. (8).

Fig. 4(a) summarizes corollaries 1 and 2. In the blue and red regions, only $R_{1}$ and $R_{2}$ experiences a reduced variance from hybridization respectively. However, in the yellow region, both sensors receive the same average power output with reduced variance. This implies that hybridization boosts exploitation of both resources. In Fig. 4(b), the variance reduction ratio 


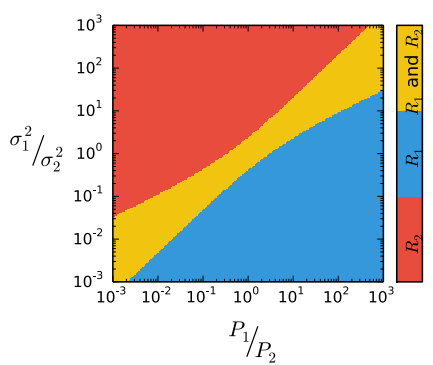

(a)

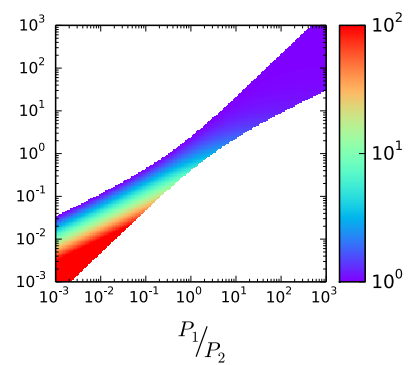

(b)
Figure 4: (a) The variance reduction map for hybridization of two resources; (b) Variance reduction ratio for $R_{1}$ in the region profitable to both.

Table II: Simulation Parameters

\begin{tabular}{l|c|c|c} 
& Symbol & $S_{1}$ & $S_{2}$ \\
\hline Energy Density (J) & $E_{d}$ & 3.0 & 0.75 \\
\hline Energy Probability & $E_{p}$ & 0.25 & 0.60 \\
\hline Storage (J) & $\mathrm{C}$ & 2000 & 2000 \\
\hline Transmission Energy (J) & $E_{t}$ & 600 & 400 \\
\hline Stand-by Energy (J) & $E_{s}$ & 0.05 & 0.04
\end{tabular}

in the yellow region for $S_{1}$ is presented. Depending on the average power outputs of the resources, a 100-fold variance reduction is possible while improving the performance of the other resource as well.

In order to test the performance of hybrid energy harvesting in a realistic communication scenario, we simulated hybridization of two sources driving two sensor nodes with a certain reporting frequency. The nodes, $S_{1}$ and $S_{2}$ can harvest $E_{d}$ amount of energy with a probability of $E_{p}$ at each second. Their storage is limited to $C$, which are initially full. They need $E_{t}$ to transmit and $E_{s}$ per second to stand-by. Unless the nodes have enough energy to stand-by, they die. In order to preserve energy to stand-by, the sensors transmit only if they have enough energy to transmit and stand-by till the next cycle. Otherwise, they skip to harvest more energy till the next transmission. Simulation parameters are presented in Table II. The simulation results are shown in Fig. 5.

From Fig. 5, we realize that for less frequent reporting, the nodes $S_{1}$ and $S_{2}$ can survive on their own without dropping reports, as they can keep their storage mostly full. However, as the frequency increases, hybrid energy harvesting outperforms single source harvesters. Due to diverse energy scavenging, the overall uncertainty reduces. Therefore, hybrid energy harvester is either able to report more frequently for a given drop rate, or it reduces the drop rate for a fixed reporting frequency.

Using Theorem 1 and its corollaries, we prove that hybridization definitely boosts performance of at least one resource by reducing its variance and in some applications, it may offer a variance reduction for all of them. We also demonstrate the effectiveness of hybrid energy harvesting for a specific communication scenario. Harvesting the same output with a reduced variance reduces the storage overflows and packet drops due to energy deficiency. Since battery powered systems have zero power variance, as the variance reduces, we further approach the battery approximation, boosting system

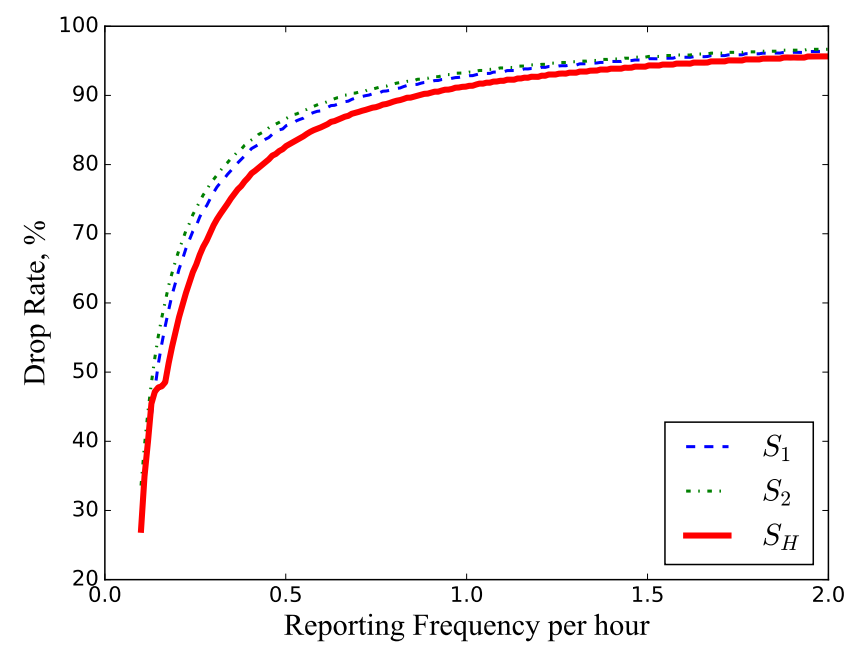

Figure 5: Drop Rate for $S_{1}, S_{2}$ and $S_{H}$ for different reporting frequencies.

reliability. Keep in mind that IoHEHT are expected to be deployed in hostile environments, where system reliability may be crucial. This makes IoHEHT a perfect candidate for Smart Cities.

\section{Energy And Data Queue}

Current energy harvesting mechanisms assume two queues: energy queue and data queue. Data queue is an inherent part of any communication system, where outgoing packages are stored in a queue to be processed as soon as the channel is available. In $\mathrm{EH}$, channel availability is no longer the only issue. Now, in order to send the packages, we also need to have enough energy to transmit them. Therefore, we need an Energy Queue. Energy queue is basically the existing energy in the system at that instant. Although it is continuous, it is assumed to be quantized where each quanta of energy is enough to transmit one package in the data queue.

The existing infinite data queue models are borrowed from generic network architectures, where all data packages are eventually processed. Using this assumption, previous works

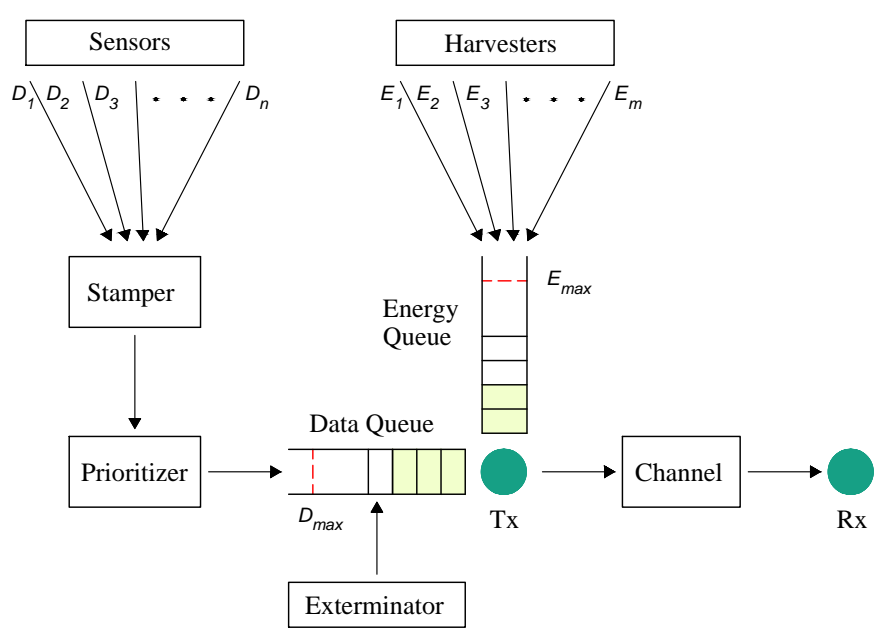

Figure 6: A hybrid energy harvesting communication model. 
optimize either transmission time $T$ of a fixed data load $B$ or vice versa [24], [25]. However, in IoHEHT, due to the extreme power constraints, assuming all packages are sent is unrealistically optimistic. Although hybrid energy harvesting improves the energy line of the system, data line may need to be improved as well. Hence, an efficient IoEHT specific data queue should include the following parts:

- Stamper: A simple circutry that adds a time and priority stamp to the incoming sensor data. Such a part is essential if multiple sensor data is transmitted through a single transmitter.

- Prioritizer: Important data is prioritized such that in case of energy shortage, less important data will be delayed or dropped. For example, out of ordinary sensor data carries a higher importance than average/ordinary sensor data. Such a circuit can be built easily by comparing a few bits to the average or expected values, where the comparator size can be adjusted depending on the system precision.

- Exterminator: Real time surveillance and monitoring are among the primary tasks of IoT Networks. If a new update from a source arrives, the previous package may lose its importance. Therefore, in case of continuing energy shortage, packages that extends a waiting period and/or packages which were outdated by the arrival of new packages may be exterminated from the data queue in order to prevent dropping newer package off the data queue and give them a better transmission probability.

Note that storing data in a fast access memory is energy consuming. Therefore, having a long data queue may not be optimal. An IoEHT specific data and energy queue is presented in Fig. 6, where $\mathrm{N}$ sensors feed the same transmitter powered by $m$ harvesters.

Combining the energy variation of hybrid energy harvesting with application and energy profile specific data queue management system similar to Fig. 6, helps us to form a feasible energy tunnel even in the most extreme energy scenarios. Therefore, using IoHEHT, with enhanced sensing and control, we can move one step closer to the Smart City concept.

\section{PRotocol Stack}

The hybrid EH method is utilized to overcome the limitations of batteries for different IoT applications. However, it has not yet been entirely applied in the domain of the IoT. Furthermore, the applications of IoT play a crucial role in the realization of Smart Cities. The existing wireless network protocols should be reconsidered to enable the IoT for Smart Cities.

Although there is number of efforts to realize IoT vision [5], [27], there has been no studies on hybrid EH in IoT domain. Proposed approaches should consider overcoming the intermittent availability of EH resources by diversifying the sources with the utilization of the hybrid approach. Combining different EH resources according to their availability offers immense flexibility. Furthermore, the vision for the Smart Cities intensifies the challenges posed by the IoT paradigm since Smart Cities have harsh environments in terms of channel and environmental conditions.

\section{A. Physical Layer}

Due to the adoption of hybrid EH approach, the physical layer in IoT enabled Smart Cities should be considered as a new design problem. The existing solutions for physical layer such as coding [28] and modulation [29] do not consider the hybrid approach and battery-free IoT operation in Smart Cities. Hence, some open issues for physical layer in IoT domain can be itemized as follows.

- The low-complexity devices in IoT requires using not high order modulation techniques but novel backscatter modulation techniques such as 4-QAM backscatter modulation [29]. This study should be modified according to the harsh environment of Smart Cities and the hybrid EH approach.

- The maximum power transmission efficiency should be studied by modeling the newly proposed hybrid $\mathrm{EH}$ method since the resource constraint of the sensor nodes is alleviated by the hybrid $\mathrm{EH}$ approach.

- A new EH scheme should be proposed to satisfy the requirements of the communication according to the harvestable resources for different IoT applications in Smart Cities. This scheme must be adaptive to take the ever changing availability of the EH resources into account.

- An efficient power management scheme considering the availabilities of the adopted EH schemes should be designed in order to support the battery-free operation of the sensor nodes in IoT enabled Smart Cities. The power management scheme improves the connectivity of the nodes due to increased harvestable energy in IoT enabled Smart Cities. The harvested energy from different resources boosts the available power for the sensor nodes, increasing the coverage and communication QoS of the sensors in Smart Cities. Harsh environment of the Smart Cities causes dynamic topology changes such as in industrial areas [26]. The advantages of the hybrid $\mathrm{EH}$ approach ease the problem of the dynamical change of the channel.

- The complexity of utilizing different EH circuitry for enabling IoHEHT should be analyzed and its effects should be studied. To this end, a detailed inspection of the harvester circuitry is necessary for energy-efficient operation of the devices in IoHEHT enabled Smart Cities.

\section{B. Data Link Layer}

Diversification of $\mathrm{EH}$ resources by the hybrid approach decreases the possibility of intermittency of captured energy. Hence, it increases the transmitted power in the long run due to more frequent arrivals of harvested energy. It increases the signal-to-noise ratio (SNR) of the received signal, which decreases the error in transmitted packets. Therefore, the existing solutions in medium access protocol [30] and error correction [31] needs to be considered to support battery-free operation in Smart Cities. Considering these facts of hybrid energy harvesting, the open issues in IoT in Smart Cities are itemized as follows.

- In order to take full advantage of hybrid EH, error control mechanisms, which are automatic repeat request (ARQ) 
and forward error correction (FEC), should be revisited according to the hybrid energy harvesting approach. Different error correcting methods may be utilized to enable efficient communication in Smart Cities.

- Power consumption and the reliability of the hybrid EH method should be investigated under different energy profiling schemes, which are online and offline schemes.

- Another challenging issue in IoHEHT domain is medium access control. The hybrid approach for $\mathrm{EH}$ increases the possibility of battery-free operation of the sensors by increasing the availability of harvestable energy continuity. To enhance the availability of continuous energy, energyefficient medium access techniques should be proposed.

- The spectrum bands are heavily utilized in urban areas [39]. It makes the access to the spectrum difficult and increases the delay. Hence, spectrum-aware solutions may be applicable in this domain to realize energy-efficient IoT enabled Smart Cities by considering the cognitive radio approaches in [32]. On the other hand, for timecritical operations, the battery-free operation may be degraded due to higher power consumption to convey the information in time. Hence, the characteristics of different $\mathrm{EH}$ resources and the application affect the design of medium access protocol.

\section{Network Layer}

Although different technologies are merged in the IoT domain, the IoT applications must support IPv6 [33]. Furthermore, device energy consumption and hop count should be taken into account for routing protocols. In IoT domain, a routing protocol for low power and lossy networks adopting IPv6 [34]. Also, the different amount of harvestable energy due to randomness exploitable resources causes a very dynamic environment for routing solutions in IoHEHT in Smart Cities. Hence, the open issues for network layer for IoHEHT should consider these issues. They are listed as follows.

- For data centric and flat architecture protocols, the nodes with more harvested energy should participate in the routing process. For instance, a node located near a light source in a city can harvest more energy than the other nodes in its neighborhood, making it suitable to become a relay node in a scenario for IoT enabled Smart Cities. Hence, proposed solutions should consider this issue in IoHEHT domain.

- In hierarchical routing algorithms, the nodes with the highest harvested energy should be the cluster-heads in their neighborhood since operation of the cluster-head node is energy-consuming due to their communication with other cluster members and inter-cluster communication. Hence, hybrid EH-aware clustering techniques should be studied.

- Location-based routing algorithms are energy-efficient algorithms, however, they require location information of the nodes, which is a difficult task for heavily deployed IoT scenarios. This problem should be studied considering the hybrid EH approach.

- The routing protocols should also consider the spatiotemporal change in the overall harvestable energy of each node in a distributed manner to increase the self sustainability of the nodes in IoT enabled Smart Cities.

\section{Transport Layer}

End-to-end reliability and congestion control are the key goals of transport layer. Transport Control Protocol (TCP) is heavily used, however, it can not support the IoT applications. Connection setup of TCP depletes battery of resource constrained IoT nodes, and congestion control of TCP would be useless due to small IoT packet sizes and challenging IoT environments [4]. Hence, new transport protocols schemes should be considered. The open issues in transport layer for IoHEHT in Smart Cities are listed as follows.

- The nodes with more harvested energy will be more active, which generate and send more packets and contribute to the congestion of the network. Hence, IoHEHTspecific congestion detection and avoidance protocols should be proposed. These protocols should be aware of the harvested energy to predict the congestion in the IoT and take measures to avoid congestion.

- In Smart Cities, there may be some hot spots contributing to the congestion of the IoT. This problem can also be overcome by spectrum-aware solutions. However, spectrum-aware solutions may be energy-consuming due to cognition operation in spectrum usage, which can be overcome by the hybrid EH approach.

- Transport protocols should be studied according to the energy and data queue specifications discussed in Section V. Also, these transport protocols should include offline or online energy profiling to better utilize diverse harvestable resources.

- In order to increase the energy efficiency of the IoT nodes, data redundancy of the IoT observations should also be considered. These observations are correlated in time and spatial domain [40]. If this correlation is manipulated, less data packets would be enough to extract the information about the observed phenomena in Smart Cities. This will provide energy-efficiency for resource constrained IoHEHT networks.

- The reliable delivery of the packets to the gateway in the IoT depends on a number of parameters, one of which is the harvested energy of the packet forwarding IoHEHT nodes. The random nature of harvested energy for each node causes a highly dynamic environment. Hence, the routing and harvested energy for the end-to-end reliability require cross-layer communication solutions, taking advantage of the higher EH in IoHEHT networks.

\section{E. Cross-Layer Design Options}

Different communication requirements among wireless devices in IoT and heterogeneity in the capabilities of them necessitate the use of cross layer solutions to support adaptive approaches [36]. Although there exists cross-layer design options for wireless sensor networks [37], [38], these solutions cannot be adopted in IoT domain. It is the case due to the heterogeneous capabilities of IoT devices, different QoS requirement of these devices, and their individual goals [36]. 
Furthermore, the IoT architectures should consider the Internet connection, however, the existing protocols [37], [38] do not consider it.

Proposed cross-layer solutions should consider the relation between different network layers to propose novel algorithms that decreases energy consumption, provide seamless Internet connectivity and satisfy desired QoS requirements. Hence, design options for cross-layer in IoHEHT for Smart Cities are listed as follows.

- Cross-layer protocols should also consider the harsh environment of Smart Cities with the hybrid EH approach. Better energy profile by hybrid $\mathrm{EH}$ and the bad channel conditions in Smart Cities requires the consideration of physical, data link layer and network layer together since joint consideration during network optimization provides better solutions [36].

- The transceiver design should also consider the continuity of the harvesting resources. This design should also consider the channel conditions to minimize errors in the channel. Hence, there is a need for a transceiver design that considers different network layers together.

- Reliable transmission control scheme based on FEC sensing is proposed in [31]. It depends on the cross-layer design to improve resource utilization and reliability in IoT domain. However, this design does not consider the challenges posed by hybrid EH and Smart Cities. Hence, an energy efficient cross-layer transmission scheme is necessary.

- The standardization of IoT devices is essential to build large networks. Such standardization may reduce the overall heterogeneity of the network, and alleviate the inefficiencies due to cross layer solutions.

\section{CONCLUSION}

Hybrid energy harvesting wireless networks are envisioned to play a key role in realizing IoT. This method paves a way for alleviating the constraints of existing harvesting methods. We believe that this study will broaden the scope of energy harvesting procedures, and make the battery-less wireless devices possible in the very near future. In this work, we surveyed different energy harvesting methods and how to combine them, in order to obtain internet of hybrid energy harvesting things. We investigated open issues in IoHEHT communications and proposed IoHEHT specific hardware. IoHEHT has the potential to completely eliminate the batteries without reducing the system performance. Moreover, thanks to the diverse energy harvesting, IoHEHT is one of the best candidates to deploy in hostile environments.

\section{REFERENCES}

[1] H. Schaffers et al., "Smart cities and the future internet: Towards cooperation framewors for open innovation," The Future Internet, Lect. Notes Comput. Sci., vol. 6656, pp 431-446, 2011.

[2] S. Chen et al., "A Vision of IoT: Applications, Challenges, and Opportunities with China Perspective," IEEE Internet of Things Journal, vol. 1, no. 4, pp. 349-359, Aug. 2014.

[3] O. Vermesan and P. Friess, "Internet of Things Strategic Research and Innovation Agenda," in Internet of Things - Converging Technologies for Smart Environments and Integrated Ecosystems, Denmark, River Publishers, 2013.
[4] L. Atzori et al., "The Internet of Things: A survey," Computer Networks, vol. 54, no. 15 , pp. 2787-2805, Oct. 2010.

[5] A. Zanella et al., "Internet of Things for Smart Cities," IEEE Internet of Things Journal, vol. 1, no. 1, pp. Feb. 2014.

[6] S. Sudevalayam, and P. Kulkarni, "Energy harvesting sensor nodes: Survey and implications," IEEE Comm. Surveys \& Tutorials, vol. 13, no. 3, pp. 443-461, Sept. 2011.

[7] J. W. Matiko et al., "Review of the application of energy harvesting in buildings," Measurement Science and Technology vol. 25, no. 1, Sept. 2013.

[8] D. Villiers et al., "Energy harvesting for a condition monitoring mote," 34th Annual Conference Industrial Electronics, IECON Nov. 2008, pp. 2161-2166.

[9] J. C.-Farrarons et al., "A multiharvested self-powered system in a lowvoltage low-power technology," IEEE Transactions on Industrial Electronics, vol. 58., no. 9, pp. 4250-4263, Sept. 2011.

[10] D. Carli et al., "An effective multi-source energy harvester for low power applications," IEEE Design, Automation \& Test in Europe, Mar. 2011, pp. 1-6.

[11] H. Li et al., "Design and Experimental Evaluation on an Advanced Multisource Energy Harvesting System for Wireless Sensor Nodes," The Scientific World Journal, June 2014.

[12] T. Kang et al., "An Energy Combiner for a Multi-Input EnergyHarvesting System," IEEE Transactions on Circuits and Systems II: Express Briefs, vol. 62, no. 9, pp. 911-915, Sept. 2015.

[13] J. Jessen et al., "Design considerations for a universal smart energy module for energy harvesting in wireless sensor networks," Proceedings of the 9th Workshop for Intelligent Solutions in Embedded Systems (WISES), Jul. 2011, pp. 35-40.

[14] F. Akhtar and M. H. Rehmani, "Energy replenishment using renewable and traditional energy resources for sustainable wireless sensor networks: A review," Renewable and Sustainable Energy Reviews, vol. 31, no. 45, pp. 769-784, May 2015.

[15] R. Moghe et al., "A scoping study of electric and magnetic field energy harvesting for wireless sensor networks in power system applications," Proc. IEEE Energy Conv. Congr. \& Expo., Sep. 2009, pp. 3550-3557.

[16] M. L. Ku, et al., "Advances in Energy Harvesting Communications: Past, Present, and Future Challenges," IEEE Communications Surveys \& Tutorials, vol. 18, no. 2, pp. 1384-1412, Nov. 2015.

[17] O. Cetinkaya and O. B. Akan, "Electric-field Energy Harvesting Wireless Networks," to appear in IEEE Wireless Communications, 2016.

[18] "R. Moghe et al., Smart "Stick-on" Sensors for the Smart Grid," IEEE Transactions on Smart Grid, vol. 3, no. 1, pp. 241-252., Mar. 2012.

[19] H. Kim et al., "Stray electric field energy harvesting technology using MEMS switch from insulated AC power line," IET Electronics Letters, vol. 50, no. 17, pp. 1236-1238, Aug. 2014.

[20] R. Moghe et al., "A Low-Cost Electric Field Energy Harvester for an MV/HV Asset-Monitoring Smart Sensor," IEEE Trans. on Ind. App., vol. 51, no. 2, pp. 1828-1836, Mar. 2015.

[21] Q. R. Xu et al., "Miniature self-powered stick-on wireless sensor node for monitoring of overhead power lines," IEEE Energy Conversion Congress and Exposition, Sept. 2013, pp. 2672-2675.

[22] H. S. Kim et al., "Power management circuit for wireless ubiquitous sensor nodes powered by scavenged energy," IET Electronics Letters, vol. 45, no. 7, pp. 373-374, Mar. 2009.

[23] J. Yang et al., "Optimal packet scheduling in an energy harvesting communication system," IEEE Transactions on Communications, vol. 60. no. 1, pp. 220-230, Jan. 2012.

[24] O. Ozel et al., "Fundamental Limits of Energy Harvesting Communications," IEEE Comm. Mag., vol. 53, no. 4, pp. 126-131, April 2015.

[25] J. Yang et. al, "Optimal Packet Scheduling in an Energy Harvesting Communication System," IEEE Trans. Comm., vol. 60, no. 1, pp. 220230, Jan. 2012

[26] V. C. Gungor and G. P. Hancke, "Industrial Wireless Sensor Networks: Challenges, Design Principles, and Technical Approaches," IEEE Transactions on Industrial Electronics, vol. 56, no. 10, pp. 4258-4265, Oct. 2009.

[27] Z. Sheng et. al, "A Survey on the IETF Protocol Suite for the Internet of Things: Standards, Challenges, and Opportunities," IEEE Wireless Communications, vol. 20, no. 6, pp. 91-98, Dec. 2013.

[28] J. Gao et. al, "RFID Coding, Name and Information Service for Internet of Things," in Proc. IEEE CCWMSN, 2007.

[29] R. Correia and N. B. Carvalho, "Design of high order modulation backscatter wireless sensor for passive IoT solutions," in Proc. IEEE Wireless Power Transfer Conference, 2016. 
[30] M. C. Vuran, I. F. Akyildiz, "Spatial correlation based collaborative medium access control in wireless sensor network," IEEE/ACM Trans Networking, vol. 14, no. 2, pp. 316-329, 2016.

[31] Y. Jin, "Reliable Transmission Control Scheme based on FEC Sensing and Adaptive MIMO for Mobile Internet of Things," Journal of Comunications, vol. 9, no. 12, Dec. 2014.

[32] M. A. Shah, S. Zhang, and C. Maple, "Cognitive radio networks for Internet of Things: Applictions, challenges and future," in Proc. IEEE ICAC, 2013.

[33] S. Deering, R. Hinden, Internet Protocol, Version 6 (IPv6) Specification, RFC 2460, December 1998.

[34] Routing over Low Power and Lossy Networks (RoLL), IETF, http://datatracker.ietf.org/wg/roll/charter.

[35] V. Cerf, Y. Dalal, C. Sunshine, Specification of Internet Transmission Control Program, IETF RFC 675, December 1974

[36] C. Han, J. M. Jornet, E. Fadel, and I. F. Akyildiz, "A cross-layer communication module for the Internet of Things," Computer Networks, vol. 57, pp. 623-633, 2013.

[37] M.C. Vuran, I.F. Akyildiz, "Xlp: a cross-layer protocol for efficient communication in wireless sensor networks," IEEE Transactions on Mobile Computing, vol. 9, no 11, (2010) 15781591.

[38] P. Park, P. Di Marco, and K. Johansson, "Cross-layer optimization for industrial control application using wireless sensor and actuator mesh networks," IEEE Transactions on Industrial Electronics, Nov. 2016.

[39] I. F. Akyildiz et. al, "NeXt generation/dynamic spectrum access/ cognitive radio wireless networks: A survey," Computer Networks, vol. 50, no. 13, pp. 2127-2159, Sep. 2006.

[40] M. C. Vuran, O. B. Akan, and I. F. Akyildiz, "Spatio-temporal correlation: theory and applications for wireless sensor networks," Computer Networks, vol. 45, no. 3, pp. 245-259, Jun. 2004. 